\title{
Waning but persistent humoral response 6 months after the third dose of the mRNA BNT162b2 vaccine in hemodialysis and peritoneal dialysis patients
}

\author{
Pierre Housset ${ }^{1} \cdot$ Sabah Kubab ${ }^{2}$ - Agathe Pardon ${ }^{1} \cdot$ Nathalie Vittoz $^{1}$. Dogan-Firat Bozman ${ }^{1} \cdot$ Latifa Hanafi $^{1}$. \\ Valérie Caudwell ${ }^{1} \cdot$ Anne-Laure Faucon ${ }^{1,3}$ (1)
}

Received: 24 December 2021 / Accepted: 6 February 2022 / Published online: 22 February 2022

(c) The Author(s) under exclusive licence to Italian Society of Nephrology 2022

Keywords SARS-CoV-2 COVID-19 · mRNA BNT162b2 vaccine $\cdot$ Humoral response $\cdot$ Hemodialysis $\cdot$ Peritoneal dialysis

Patients receiving maintenance dialysis have a diminished humoral response to SARS-CoV-2 vaccination, which led the French National Authority for Health to recommend, in April 2021, a third dose in the first set of vaccination in this population. If the third vaccine dose substantially increased antibody $(\mathrm{Ab})$ levels in dialysis patients [1-3], the longterm durability and the robustness of a protective humoral response against SARS-CoV-2 remains unknown.

We assessed the dynamics of the humoral response of both hemodialysis (HD) and peritoneal dialysis (PD) patients from the Nephrology Department of the Centre Hospitalier Sud-Francilien (Corbeil-Essonnes, France), before and at one (M1), three (M3) and six (M6) months after a third dose of the mRNA BNT162b2 vaccine (Pfizer-BioNTech ${ }^{\circledR}$ ). Patients with a history of symptomatic COVID-19 or with asymptomatic COVID-19 after the third dose, patients without serology results at M1 or M6, and those who received a kidney transplant, or died were excluded (Figure S1).

Humoral response was evaluated by measuring the plasma concentrations of anti-SARS-CoV-2 spike protein $\mathrm{S} 1$ total immunoglobulin antibodies using the Roche

Pierre Housset

pierre.housset@chsf.fr

$\triangle$ Anne-Laure Faucon

anne-laure.faucon@chsf.fr

1 Department of Nephrology, Centre Hospitalier SudFrancilien, Corbeil-Essonnes, France

2 Department of Microbiology, Centre Hospitalier Sud-Francilien, Corbeil-Essonnes, France

3 INSERM UMRS 1018, Clinical Epidemiology Unit, Centre for Research in Epidemiology and Population Health, Paris-Saclay University, Villejuif, France
Elecsys ${ }^{\circledR}$ immunoassay [4]. According to the manufacturer's protocol, patients with an anti-spike antibody titer below $0.8 \mathrm{AU} / \mathrm{mL}$ were classified as seronegative. An anti-spike antibody titer below $257 \mathrm{AU} / \mathrm{mL}$-corresponding to the threshold of the WHO International standard unit of 264 binding antibody units [BAU]/mL[5] which offers $80 \%$ protection against symptomatic COVID-19 [6]—was classified as a low antibody titer. The kinetics of the humoral response was assessed as the ratio of the difference in anti-spike antibody titer between M1 and M6, over the titer at M1, and expressed in percentage. Clinical and biological data were collected as previously detailed [1]. Anti-nucleocapsid serology was also systematically performed before the third vaccine dose. Wilcoxon and Fisher's exact tests were used to compare quantitative and qualitative variables, respectively. Wilcoxon signed rank test was used to analyze the paired data. Statistical analyses were conducted using $R^{\circledR} 3.6$ and GraphPad Prism ${ }^{\circledR}$ softwares.

Sixty-eight patients ( $n=34 \mathrm{HD}$ and $n=34 \mathrm{PD})$ were included, with a dialysis vintage of 3.0 [interquartile range, IQR: $1.0 ; 6.0]$ years (Table $\mathrm{S} 1$ ). Median age was 66.0 [53.8; 76.3 ] years, $65 \%$ were men and $18 \%$ had a history of immunosuppression. After 6-month follow-up, the anti-spike antibody titer significantly decreased from 6924 [1903; 11485] AU/mL at M1, to 2035 [597; 4009] $\mathrm{AU} / \mathrm{mL}$ at M3, and to $875[290 ; 1979] \mathrm{AU} / \mathrm{mL}$ at M6 $(p<0.0001)$ (Figs. 1 , $\mathrm{S} 2$ ), corresponding to a median decrease in anti-spike antibody titer of 84.3\% [75.5, 88.0] between M1 and M6.

The rate of patients with a low antibody titer (i.e., $<257 \mathrm{AU} / \mathrm{mL}$ ) increased from $8.8 \%$ at M1 to $25 \%$ at M6, but no patient sero-reverted with 67 seropositive patients (98.5\%) at M6. Compared with patients with an antibody titer $\geq 257 \mathrm{AU} / \mathrm{mL}$ at 6 months, those with an antibody titer $<257 \mathrm{AU} / \mathrm{mL}$ were older, were more likely to have 


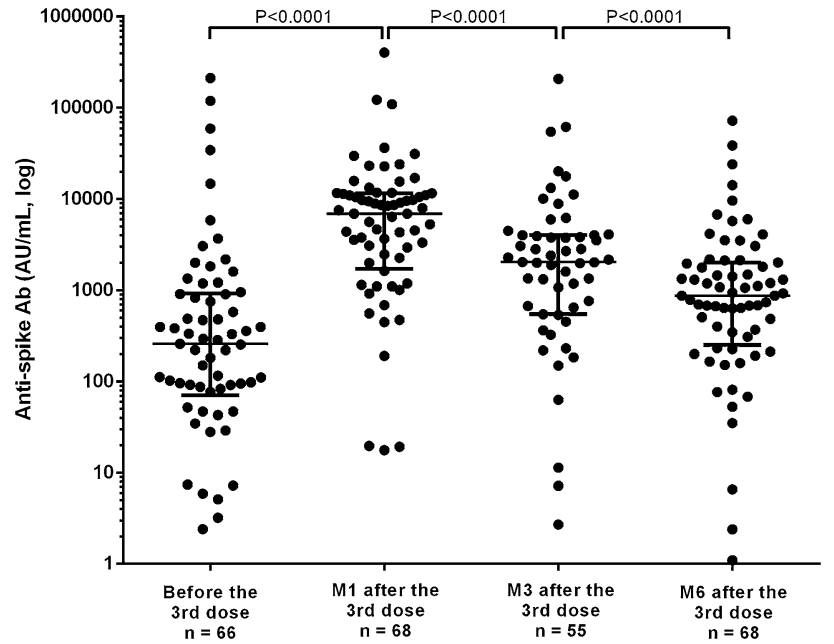

Fig. 1 Kinetics of anti-spike antibodies before and after the third vaccine dose. Figure shows the anti-spike antibody levels before, and one, three and 6 months after the third dose of the mRNA BNT162b2 vaccine in dialysis patients. Each point represents individual data. Antibody titers lower than 1 could not be plotted in the graph because of logarithm scale. Horizontal lines indicate the median with interquartile range. The Wilcoxon signed rank test was used to analyze the paired data

a history of immunosuppression, had a lower antibody titer before and one month after the third vaccine dose, and lower serum gamma globulin and albumin levels (Table S1). Humoral response was not different between HD and PD patients (Table S2). Patients with positive anti-nucleocapsid serology incidentally found before vaccination, had a higher titer and a lower decrease in Ab titer at M6 (Table S3).

This is the first description of the 6-month kinetics of humoral response after the third vaccine dose against SARS$\mathrm{CoV}-2$ in dialysis patients. Our data show a waning humoral response over time, with a median decrease in antibody titer of $84.3 \%$ in 6 months and a rate of patients with low antibody titer increasing from 8.8 to $25 \%$ between M1 and M6. However, median anti-spike antibody titer remained more than threefold higher 6 months after than before the third dose, with a seropositivity rate of $98.5 \%$, and no seroreversion, or symptomatic COVID-19. Studies assessing the humoral response after a two-dose regimen in dialysis patients showed a sero-reversion rate of $5.8 \%$ at 3 months [7] and 32\% at 6 months [8]. This confirms the need for a three-dose regimen and further suggests that a fourth boost dose should be considered in this population to increase both breadth and cross-reactivity of neutralizing antibodies, given the potential of the SARS-CoV-2 to escape vaccine-induced humoral response [9].

Study limitations include small sample size, a limited follow-up of 6 months, and lack of cellular immunity testing and neutralizing antibody testing.
To conclude, our results show that after a three-dose regimen of the BNT162b2 vaccine, antibody titer decreased over time with a significant number of dialysis patients with a low antibody titer, even if no sero-reversion or symptomatic COVID-19 occurred at 6 months. Given the pandemic's ongoing waves of new infections, a fourth boost dose should be proposed to obtain a sustained protective humoral response in this population.

Supplementary Information The online version contains supplementary material available at https://doi.org/10.1007/s40620-022-01276-2.

Acknowledgements The authors thank the patients for their participation in the study.

Author contributions Research idea and study design: PH, ALF; data acquisition: $\mathrm{PH}, \mathrm{ALF}$; anti-spike serology testing: SK; data analysis and interpretation: PH, ALF; statistical analysis: ALF; supervision or mentorship: PH, ALF. Each author contributed important intellectual content during manuscript drafting or revision.

Funding None.

Data availability The data that support the findings of this study are available from the corresponding author upon reasonable request.

\section{Declarations}

Conflict of interest None.

Disclosures None.

Ethics approval and informed consent According to French law (Loi Jardé), anonymous retrospective studies do not require institutional review board approval.

\section{References}

1. Bensouna I, Caudwell V, Kubab S et al (2021) SARS-CoV-2 antibody response after a third dose of the BNT162b2 vaccine in patients receiving maintenance hemodialysis or peritoneal dialysis. Am J Kidney Dis. https://doi.org/10.1053/j.ajkd.2021.08.005

2. Ducloux D, Colladant M, Chabannes $M$ et al (2021) Humoral response after 3 doses of the BNT162b2 mRNA COVID-19 vaccine in patients on hemodialysis. Kidney Int 100:702-704. https:// doi.org/10.1016/j.kint.2021.06.025

3. Robert T, Lano G, Giot M et al (2021) Humoral response after SARS-COV2 vaccination in patient undergoing maintenance hemodialysis: loss of immunity, third dose and non-responders. Nephrol Dial Transplant. https://doi.org/10.1093/ndt/gfab299

4. Muench P, Jochum S, Wenderoth V et al (2020) Development and validation of the elecsys anti-SARS-CoV-2 immunoassay as a highly specific tool for determining past exposure to SARSCoV-2. J Clin Microbiol 58:e01694-e1720. https://doi.org/10. 1128/JCM.01694-20

5. Infantino M, Pieri M, Nuccetelli M et al (2021) The WHO International Standard for COVID-19 serological tests: towards harmonization of anti-spike assays. Int Immunopharmacol 100:108095. https://doi.org/10.1016/j.intimp.2021.108095 
6. Feng S, Phillips DJ, White T et al (2021) Correlates of protection against symptomatic and asymptomatic SARS-CoV-2 infection. Nat Med 27:2032-2040. https://doi.org/10.1038/ s41591-021-01540-1

7. Broseta JJ, Rodríguez-Espinosa D, Bedini JL et al (2021) Antibody maintenance 3 months after complete messenger RNA COVID-19 vaccination in haemodialysis patients. Nephrol Dial Transplant. https://doi.org/10.1093/ndt/gfab272

8. Davidovic T, Schimpf J, Abbassi-Nik A et al (2021) Waning humoral response 6 months after SARS-CoV-2 vaccination with the mRNA-BNT162b2 vaccine in hemodialysis patients: time for a boost. Kidney Int 100:1334-1335. https://doi.org/10.1016/j.kint. 2021.10.006
9. Garcia-Beltran WF, St Denis KJ, Hoelzemer A et al (2022) mRNA-based COVID-19 vaccine boosters induce neutralizing immunity against SARS-CoV-2 omicron variant. Cell S00928674(21):01496-01503. https://doi.org/10.1016/j.cell.2021.12. 033

Publisher's Note Springer Nature remains neutral with regard to jurisdictional claims in published maps and institutional affiliations. 